\title{
EnerPort: Irish Blockchain project for peer- to-peer energy trading
}

\section{An overview of the project and expected contributions}

\author{
Piyush Verma ${ }^{1 *} \mathbb{D}$, Brian O'Regan ${ }^{1}$, Barry Hayes ${ }^{2,3}$, Subhasis Thakur ${ }^{3}$ and John G. Breslin ${ }^{3}$
}

\author{
* Correspondence: piyush.verma@ \\ ierc.ie \\ ${ }^{1}$ International Energy Research \\ Centre, Cork, Ireland \\ Full list of author information is \\ available at the end of the article
}

\begin{abstract}
Executive summary
"EnerPort" is an Irish government- and industry-funded collaborative project where blockchain technology is being used to develop a peer-to-peer (P2P) energy trade model to support energy trade between microgrids. This project is led by the International Energy Research Centre partnering with National University of Ireland Galway and University College Cork, and aims to address some key challenges around associated hardware and software requirements, as well as analysing market and regulatory issues. It is a 12 month project that started on 1st March 2018 and will end on 28th February 2019. This brief report aims to introduce the EnerPort project to a broader international audience and explain all of the key details including the structure and objectives of the project, the methodology, demonstration approach, and expected results. A final report will be published at the end of the project to summarize the project results, future impact, and future work.
\end{abstract}

\section{Background}

The global electricity market is facing the disruptive 3D's: digitalization, decentralization and decarbonization which brings with it enormous changes and challenges to the electricity sector. These 3D's are not only driving the electricity consumers or businesses and industries involved in the electricity sector, but it has also made various other stakeholders beyond the electricity sector think about addressing the intersection of technology, software, economics, markets, regulations, etc. Along with these 3D's as disruptive drivers, other drivers for change are also important in terms of international and regional commitments and national plans and policies such as the Energy Efficiency Directive, the European Renewable Energy Directive, the Energy Union Package and the resulting National Plan including the Low-Carbon White Paper (Department of Communication Climate Action and Environment, 2015), the National Energy Efficiency Action Plan (Department of Communication Climate Action and Environment, 2014) and the National Mitigation Plan (Department of Communications Climate Action and Environment, 2017).

In parallel with these drivers, the penetration of renewables in Ireland has increased to $27.8 \%$ of gross electricity consumption in 2015 (Sustainable Energy Authority of Ireland (SEAI), 2016) which is increasing year-on-year, and these renewables are also

(c) The Author(s). 2018 Open Access This article is distributed under the terms of the Creative Commons Attribution 4.0 International License (http://creativecommons.org/licenses/by/4.0/), which permits unrestricted use, distribution, and reproduction in any medium, provided you give appropriate credit to the original author(s) and the source, provide a link to the Creative Commons license, and indicate if changes were made. 
being extended in terms of distributed generation/microgrids along with centralized renewable-based electricity generation. This proliferation of distributed generation enables infrastructure changes to address the dynamic nature of the demand side. Irish government is also implementing the smart meter programme which will make the electricity infrastructure more flexible to integrate with other innovative technologies. With intelligent, connected components plugged into an increasingly smarter grid, electricity consumers will become direct participants in the energy network.

Energy trading within an individual microgrid and/or between multiple microgrids is necessary to maximize the usage of renewable energy. Lack of secure, regulated and flexible markets for energy trading is the main motivation of this project. The desirable properties of such a market are (a) autonomous execution of trading decisions - autonomous execution of trading decisions will remove the burden to study the energy market and decision making of microgrid owners, (b) security of information shared for trading - security of information requires guarantees that information will not be overwritten, (c) governance of the market - governance requires checking the compliance of traders, and (d) scalable execution of market models - scalability of the market is indicated as an increase in the number of trades as the market grows. This project will use blockchain technology to deliver these properties more efficiently and securely (PricewaterhouseCoopers, 2016). Blockchain smart contracts will be used to automate the trading decision making process. A blockchain-distributed consensus protocol is a solution for the self-regulation of such a market, i.e., a few microgrids cannot deviate from the regulations of the market. Blockchain will facilitate parallel and asynchronous trades between microgrids to improve the market scalability.

This project is named as "EnerPort" and it is an industry-led collaborative research project that will investigate new market models for peer-to-peer (P2P) energy trading. ${ }^{1}$ This involves implementing blockchain technology with smart metering, and designing and demonstrating residential hardware and software platforms in the laboratory of the Power Electronics Research Centre (PERC) at NUI Galway and University College Cork. It will develop a new software tool for the co-simulation of electricity distribution networks and blockchain-based peer-to-peer energy trading. A simple block diagram to highlight the key aspects of the EnerPort project has been shown in the Fig. 1 below:

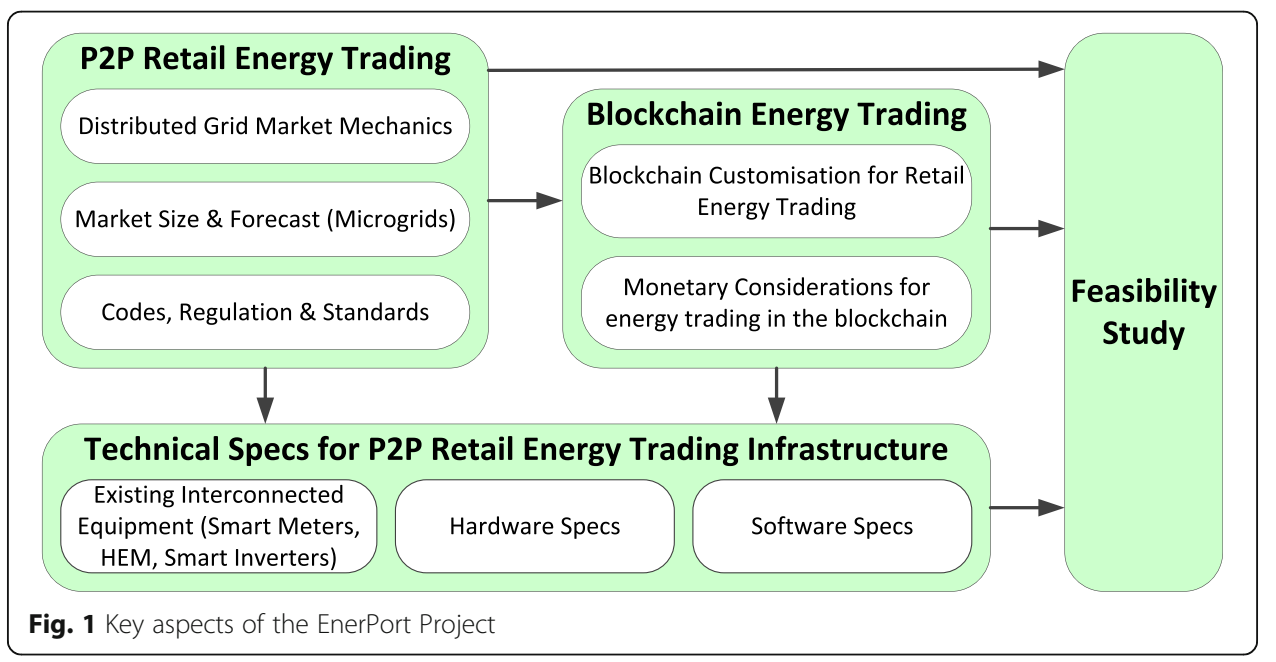


The International Energy Research Centre (IERC) is the lead member of the EnerPort consortium that includes the Insight SFI Centre for Data Analytics at NUI Galway, University College Cork and Irish companies including Systemlink, mSemicon, Verbatm.

\section{Project key objectives}

The key objectives of this project are outlined below:

\section{Market models for peer-to-peer energy trade}

- Theoretical development of double auctions, coalition formation and non-cooperative games for peer-to-peer energy trade.

- Integration of a blockchain simulator with OpenDSS (Open Source Distribution System Simulator) for an energy distribution network (McDermott, 2006; Ketter et al., 2013).

- Implementation of the blockchain system's interaction with the Open Energy Monitor platform (Open energy Monitor Platform, 2017) (on smart meters).

\section{Design and bench demonstration of residential hardware and software platforms}

- Demonstrate a functional interface between the blockchain simulator and residential smart metering and smart appliances.

- Develop a smart home bench demonstration in the Power Electronics Research Centre laboratory at NUI Galway.

- Create use cases which demonstrate the triggering of a blockchain-based P2P energy trade based on measurements from a home smart meter in order to carry out a full end-to-end simulation with hardware in the loop.

\section{Co-simulation of blockchain transactions and electricity networks}

- Develop a new software tool for the co-simulation of electricity distribution networks and blockchain-based peer-to-peer energy trading.

- Analysis of the electricity distribution network impacts of P2P energy trading in a typical LV residential distribution network.

- Release of a co-simulation tool under a suitable open license and publication of one international conference and one international journal paper disseminating these results.

\section{Advantages of using blockchain over existing solutions for P2P energy trading}

Current electricity markets are not decentralized or flexible enough to integrate massive numbers of highly-distributed variable renewable resources in an efficient manner. This problem is addressed by P2P trading, since it enables large numbers of small-scale energy prosumers to match supply/demand at a local level. This has the potential to reduce the need for system operators to provide power system flexibility and 
balancing services through conventional means (spinning and non-spinning generation reserves from large fossil-fuel power plants).

Blockchain technology is particularly suitable for P2P trading as it provides a range of benefits over any other ledger system. Securities around the system, immutability of transactions and lack of a need for an always-online system, and trusted third party to manage transactions are the main arguments of using blockchain technology. Key advantages have been explained below:

- Ease of payment: Smart contract based payment for peer to peer trade shorten the time to receive payment for energy sell and makes it easy for the common consumers.

- Ease of monetizing distributed energy generators: Quick payments, low fee for payment for energy sale with blockchain improve the potential for monetizing distributed energy generators. Blockchain makes energy generators (prosumers) such as houses and businesses as a source of additional revenue.

- No 3rd Party: There is no third party in the transaction as the value is digitally transferred from one account to another on a blockchain platform. There is a trust towards the underlying blockchain system and not on any physical third party. Lack of third party erases the concerns of trust and associated fees.

- Assured payment: Smart contract can guarantee payment for energy sell. Prosumers enter the market with smart contracts and they fund the smart contract irrespective of whether they will buy or sell. Smart contracts will be triggered with energy usage data and hence it is guaranteed that prosumers who sells energy will get paid.

- Distributed computation: The computation problem for prescribed energy usage based on energy to be bought or sold requests can be solved with distributed algorithms. It improves the robustness of the computing infrastructure.

- Enforcement of trade decisions: Trade decisions in a peer to peer trade states how much energy a peer should consume for the next round. Penalties for deviating from the trade decisions can be incorporated into the smart contracts and blockchain will ensure execution of such penalties. Thus enforcement of trade decisions can be achieved with blockchain. It will encourage peers to comply with the prescribed energy usage.

- Cost of computation: Blockchain will attract investments into building computing infrastructure for blockchain dedicated to facilitate energy trading.

- Associated services: A blockchain supported energy trade platform will support services from the blockchain for energy related applications. For example, it can be used for supporting operations in electric vehicle charging stations. Blockchain will increase interoperability among trade platforms (i.e., peer to peer energy trade from a prosumer to the charging station and the energy trade between the EV and the charging station) by bringing them to the same platform (they all can be part of the same blockchain or related blockchain using sidechains).

- Computation overhead: A concern with using blockchain for energy trade is its computation overhead which diminishes its scalability. Security of blockchain comes from data replication as originally it is not designed for scalability. But recent advancements in offline channels can improve scalability of blockchain to meet the requirement for large scale peer to peer energy trade where majority of the 
transactions among peers can be kept offline and the blockchain will not be updated frequently.

There are some start-ups which have considered the potential of blockchain technology and trying to revolutionize how energy can be generated and traded locally. Blockchain enabled peer-to-peer electricity trading by Powerledger (PowerLedger, n.d.), community powered microgrid by Brooklyn microgrid (Brooklyn microgrid, n.d.) and community energy trading platform by Powerpeers (Powerpeers community energy trading platform, n.d.) are few examples that are currently implementing demonstration projects and finding the possible ways to scale the technology. A recent research has also implemented to facilitate machine-to-machine (M2M) interactions and establish a M2M electricity market in a chemical industry where they have presented a scenario which includes an electricity consumer and two electricity producers trading with each other over a blockchain platform (Sikorski et al., 2017).

In spite of several advantages, blockchain still has number of concerns around system complexity, computational overhead, data storage, processing times etc. which will evolve over the time as the technology gets matured.

\section{Project deliverables}

Trade models

EnerPort will develop multiple trade models suitable for various scenarios of peer-to-peer energy trade.

- Coalition formation for energy trade: This trade model is suitable for cooperative peers who coordinate their energy consumption in order to maximize the overall valuation of their coalitions. A coalition structure is a partition over the peers where peers in each coalition trade energy among themselves. In (Thakur \& Breslin, 2018) an algorithm for generating hierarchical coalition structures is developed using blockchain.

- Double auction for energy trade: This trade model is designed for competitive peers where the peers participate in a double auction for energy trade. The trade model is implemented using blockchain in (Thakur et al., 2018). In this trade model, each peer submits its reservation price based on its forecast about its energy generation and consumption. Double auction determines the price of the energy trade and peers who will trade.

- Stackelberg Game for energy trade: This trade model suits a competitive environment where each peer wants to maximize its own utility from participating in the peer-to-peer energy trade. This trade involves a leader and a set of followers. The leader announces the price for energy to be bought or sold based on the announced forecast of each peer's energy generation and consumption. The followers observe such prices and decide on energy consumption accordingly.

\section{Simulation}

EnerPort will develop a new software tool for the co-simulation of electricity distribution networks and blockchain-based peer-to-peer energy trading. 
In order for peer-to-peer energy trading to gain acceptance on a large scale, it will be necessary for network operators to have the capability to model impacts on the distribution networks, and the potential effects on network performance and reliability.

This co-simulation tool is introduced in (Hayes et al., 2018). Its main features are summarised below:

- The co-simulation features a blockchain simulation in Python using agent-based modelling. Each peer of the peer-to-peer energy trade market forms a node of the blockchain peer-to-peer network. Activities of a peer acting as a blockchain node and energy trade agent are modelled using asynchronous processes. The trade models developed in (Thakur \& Breslin, 2018; Thakur et al., 2018) are implemented in the workflow of each peer.

- Detailed, three-phase modelling and simulation of medium- and low-voltage electricity networks will be carried out in the open-source electricity network simulator OpenDSS using test case networks which are representative of typical Irish distribution networks.

- The co-simulation tool provides a means of assessing the feasibility of large-scale adoption of peer-to-peer energy trading schemes, analysing their impacts on the distribution network, and validating their potential benefits. This can be used to analyse the impacts of P2P energy trading on network power flows and voltages. This will be used to analyse the long-term impacts of various P2P energy trading mechanisms on network utilisation, power quality, and overall distribution system planning and operation.

\section{Demonstration}

EnerPort will develop a demonstrator to showcase the technology. This will enable a full end-to-end demonstration of P2P energy trading in a residential home use case, using real electricity smart meter hardware in the loop. This use case will demonstrate a smart home configuration with the software and hardware infrastructure required to enable P2P blockchain energy trading. This consists of:

- A laboratory bench demonstration designed to test the required software interfaces and communications protocols, considering the requirements around data security and privacy.

- An interface between the smart metering hardware and the blockchain-based P2P energy trading simulator.

- Software for permissioned blockchain using Hyperledger and software for various functionalities of the trade models.

\section{Analysis of markets and future scenarios}

Peer-to-peer energy trading is inherently suited to certain niche applications, such as isolated microgrids and community-owned local energy infrastructure. However, it is unclear how utility-owned network infrastructure could be used to facilitate peer-to-peer energy trades, when these transactions are subject to grid regulations and requirements, and research in this area is still at a very early stage. Blockchain will need 
to compete with existing energy trading platforms (which are already automated and fast/secure) and may face the same difficulties as current aggregator services.

Accordingly, this project will examine the Irish electricity market and review the technical, economic and regulatory barriers to the adoption of P2P blockchain-based energy trading on a wide scale. In order to analyse the potential for the development of new energy services, it will present a case study of a blockchain-based electricity service operating in the Irish market. There is a significant requirement for flexibility in the Irish electricity market and in many other European energy markets, due to the high penetration of various renewable resources. It is expected that there will be significant future growth in new demand-side energy services to provide reserve, ramping, and voltage/frequency control services, and distributed blockchain-based trading may have a role to play in this. This case study will directly address these issues by examining the viability of such energy services being delivered via blockchain-based trades and report on the expected competitiveness of these services.

\section{Project partners}

This project consortium is made up of five partners where there are two Research Performing Organizations (RPOs) and three Small-to-Medium Enterprises (SMEs). Details of the organizations are available in the table below:

\begin{tabular}{|c|c|c|}
\hline Name of Project Partners & Type of organization & Expertise Contribution \\
\hline $\begin{array}{l}\text { International Energy } \\
\text { Research Centre }\end{array}$ & $\begin{array}{l}\text { RPO and Project } \\
\text { Management }\end{array}$ & $\begin{array}{l}\text { Project management, energy market analysis, policy } \\
\text { and regulations }\end{array}$ \\
\hline $\begin{array}{l}\text { National University of } \\
\text { Ireland Galway }\end{array}$ & RPO & $\begin{array}{l}\text { Software engineering, communication protocols, } \\
\text { and market design }\end{array}$ \\
\hline University College Cork & RPO & Power engineering, smart grids \\
\hline mSemicon & SME & Hardware requirements \\
\hline Systemlink Technologies & SME & Hardware requirements \\
\hline Verbatm & SME & Blockchain applications \\
\hline
\end{tabular}

A project management structure has been adopted to define and control the responsibilities, as well as for the timely and cost-effective delivery of this project. This project is led by a Project Steering Committee which consists of members from IERC, NUI Galway, University College Cork and a representative from each of the three industry partners.

\section{Conclusions}

There are several new business models being developed around the concept of prosumers (energy consumers who will also act as energy producers), but key challenges faced by these new business models still exist, including ensuring a consistent supply of energy, securing the transactions, and revenue distribution. This project aims to uniquely consider a range of trade models such as auctions, coalition formation, and other non-cooperative games to fit the case of energy trade and which must satisfy the physical aspects of energy distribution. The expected results of EnerPort are as follows:

- Trade models for peer-to-peer energy trade. 
- Simulation of peer-to-peer energy trade using blockchain.

- Demonstrator of the technology using Hyperledger fabric blockchain and smart meters.

Currently, there are very few existing projects worldwide which have been able to demonstrate the hardware and software requirements for blockchain-enabled peerto-peer energy trading and its applications in the context of a distributed grid. This project will demonstrate the hardware and software required to implement peer-to-peer energy trading in the distributed grid in a laboratory environment and through extensive blockchain simulations. This will allow industry to test peer-to-peer energy trading applications to better understand the future potential and applications of the technology in the energy domain.

This project will provide scalable proof-of-concept demonstrations for blockchainbased peer-to-peer energy trading in the distributed grid, and will address key challenges around hardware and software requirements and protocols, as well as issues around markets, regulations and policy. This will help to accelerate the deployment of novel energy trading systems and drive innovation in distributed grid technologies in Ireland, and it is anticipated that the results of this project will help to accelerate the development and deployment of this technology.

\section{Project outreach}

Get more details and regular updates about the project through the following outreach channels:

- Website o https://iercdevsrv.tyndall.ie/enerport/

- ResearchGate o https://www.researchgate.net/project/EnerPort-Blockchain-Energy-Trading

- Twitter o https://twitter.com/enerport_ie

- LinkedIn o https://www.linkedin.com/in/enerport-project-3a4709164/

\section{Endnotes}

${ }^{1} \mathrm{P} 2 \mathrm{P}$ energy trading is an application that employs energy resources in a decentralized manner, rather than in a centralized manner and further facilitates the trading of energy among different prosumers. A P2P concept for power systems was first proposed in 2007 (Beitollahi \& Deconinck, 2007).

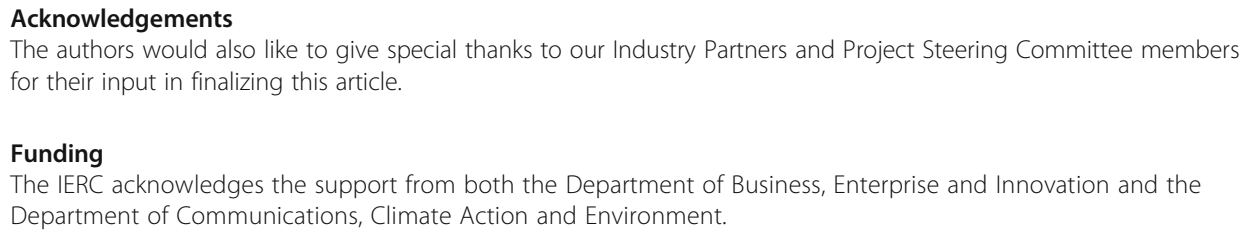


Authors' contributions

PV has drafted this overall report for an international audience. BOR has contributed in writing and finalizing the key objectives of this project. BH and ST have contributed in drafting and finalizing the deliverables of this project. JB conceived of this project idea, and contributed in finalizing this overall report. All authors read and approved the final manuscript.

\section{Competing interests}

The authors declare that they have no competing interests.

\section{Publisher's Note}

Springer Nature remains neutral with regard to jurisdictional claims in published maps and institutional affiliations.

\section{Author details}

${ }^{1}$ International Energy Research Centre, Cork, Ireland. ${ }^{2}$ University College Cork, Cork, Ireland. ${ }^{3}$ National University of Ireland Galway, Galway, Ireland.

Received: 10 July 2018 Accepted: 4 September 2018

Published online: 14 September 2018

\section{References}

Beitollahi H, Deconinck G (2007) Peer-to-peer networks applied to power grid. In: Proceedings of the international conference on risks and security of internet and systems (CRiSIS)

Brooklyn microgrid (n.d.) Available: https://www.brooklyn.energy/. Accessed 27 Aug 2018

Department of Communication Climate Action and Environment (2014) National Energy Efficiency Action Plan. DCCAE, Dublin

Department of Communication Climate Action and Environment (2015) Ireland's transition to a low-carbon community. DCCAE, Dublin

Department of Communications Climate Action and Environment (2017) National Mitigation Plan. DCCAE, Dublin

Hayes, B.P., Thakur, S., \& Breslin J.G., "Co-simulation of electricity distribution networks and Blockchain energy trading platforms", to be presented at the 11th Mediterranean conference on power generation, Transmission, Distribution and Energy Conversion (MEDPOWER) 2018

Ketter W, Collins J, Reddy P (2013) Power TAC: a competitive economic simulation of the smart grid. Energy Econ 39:262-270 McDermott TE (2006) An open source distribution system simulator, 2006. IEEE Power Engineering Society General Meeting, Montreal, Que, p 4. https://doi.org/10.1109/PES.2006.1709525

Open energy Monitor Platform, 2017, https://openenergymonitor.org/. Accessed 27 Aug 2018

PowerLedger: (n.d.) A blockchain-based peer-to-peer energy trading platform. Available: https://powerledger.io/. Accessed 27 Aug 2018

Powerpeers community energy trading platform (n.d.) Available: https://powerpeers.nl/. Accessed 27 Aug 2018

PricewaterhouseCoopers, Blockchain - an opportunity for energy producers and consumers?, 2016, URL https:/www.pwc $\mathrm{com} / \mathrm{gx} / \mathrm{en} / \mathrm{industries/assets/pwc-blockchain-opportunity-for-energy-producers-and-consumers.pdf}$

Sikorski JJ, Haughton J, Kraft M (2017) Blockchain technology in the chemical industry: machine-to-machine electricity market. Appl Energy 195:234-246

Sustainable Energy Authority of Ireland (SEAI) (2016) Energy in Ireland. SEAl, Dublin

Thakur S, Breslin JG (2018) Peer to peer energy trade among microgrids using Blockchain based distributed coalition formation method. Technol Econ Smart Grids Sustain Energy 3(5) https://doi.org/10.1007/s40866-018-0044-y

Thakur, S., Hayes, B.P., \& Breslin J.G., "Distributed double auction for peer to peer energy trade using blockchain", to be presented at the 5th international symposium on environment friendly energies and applications (EFEA) 2018

\section{Submit your manuscript to a SpringerOpen ${ }^{\circ}$ journal and benefit from:}

- Convenient online submission

- Rigorous peer review

- Open access: articles freely available online

- High visibility within the field

- Retaining the copyright to your article

Submit your next manuscript at $\boldsymbol{\nabla}$ springeropen.com 